\title{
Análise da ocorrência de downbursts no Brasil
}

\author{
Analysis of the downbursts occurrence in Brazil \\ Elias Galvan de Lima ${ }^{1}$ e Acir Mércio Loredo-Souza ${ }^{1}$ \\ ${ }^{1}$ Programa de Pós-graduação em Engenharia Civil, UFRGS
}

\begin{abstract}
Resumo
Downbursts são fenômenos meteorológicos formados a partir de tempestades convectivas severas e dão origem a intensas rajadas de vento. Quando há intenso entranhamento de ar seco e frio, em altos níveis em meio às nuvens de profundo desenvolvimento vertical, ocorre um fenômeno denominado resfriamento evaporativo, que dá origem a massas de ar densas e frias dentro da nuvem. Essas massas se mantêm sustentadas pelas correntes convectivas até o momento em que ocorrem as forças descendentes que superam as forças ascendentes, nesse momento surgem correntes que descendem até encontrar a superfície e então espalham-se horizontalmente de forma anelar. Assim, este estudo tem por objetivo analisar a ocorrência desse fenômeno no sul do Brasil por meio das características que definem a ocorrência de downbursts sugeridas pela literatura. Devido a importância do conhecimento de ambientes favoráveis a ocorrência de downbursts, procurou-se analisar também a influência dos índices de Energia Potencial Convectiva Disponível (CAPE) e Índice de Inibição Convectiva (CINE), na definição da ocorrência de ventos gerados por downbursts. Os resultados mostram que a utilização dos índices de CAPE e CINE, de forma isolada na definição de ambientes convectivos favoráveis à ocorrência de downbursts, não são suficientes e sugerese a observação de outras variáveis e indices meteorológicos nesse processo. Através de dois estudos de casos, observaram-se ventos provenientes de tempestades com características daqueles provenientes de downbursts, observando-se assim a ocorrência do fenômeno na Região Sul do Brasil.
\end{abstract}

Palavras-chave: Downburst. CAPE. CINE. Tempo severo.

\begin{abstract}
Downbursts are meteorological phenomena formed from severe convective storms and give rise to strong gusts of wind. When there is intense entrainment of dry and cold air at high levels through the clouds of deep vertical development it is established a phenomenon called evaporative cooling. This phenomenon rise up masses of dense and cold air inside the cloud that keeps sustained by convective currents until the moment that the downward forces outweigh the upward forces. At this moment streams of descend air called downdrafts are established, they fall to the ground and then spread horizontally on a ring shape. This study aims to analyze the occurrence of this phenomenon in the southern Brazil through the characteristics that define the occurrence of downbursts suggested in the literature. Because of the importance of knowledge of the occurrence of environments in which downbursts are favorable to occur, it is analyzed the influence of index Convective Available Potential Energy (CAPE) and Convective Inhibition Index (CINE) on the occurrence of winds generated by downbursts. The results show that the use of indices CAPE and CINE on an isolated form in the definition of environments favorable to the occurrence of convective downbursts are not sufficient and is suggest the observation of other meteorological variables and index on this process. Through two case studies, it was observed winds from storms with characteristics of downbursts, thus it is observed the occurrence of the phenomenon in southern Brazil.
\end{abstract}

Keywords: Downburst. CAPE. CINE. Severe weather. 


\section{Introdução}

As perdas de vidas e danos econômicos ocasionados por fenômenos meteorológicos severos estão cada vez mais expressivas e podem estar ocorrendo devido às variações ou oscilações climáticas, em consequência da expansão desenfreada dos limites urbanos às áreas de risco, do crescimento populacional expressivo, entre outros fatores.

Através de dados provenientes do Emergency Events Database (EM-DAT), Marcelino et al. (2005) mostram que, no período de 1900-2006, as causas mais frequentes para registros de desastres naturais no mundo foram inundações ou tempestades severas.

Guha-sapir (2013), destacam que é possível perceber uma tendência no aumento de desastres relacionados ao clima a nível mundial e nos danos gerados à economia devido a esses eventos para o período entre 1950 e 2012.

Segundo a defesa civil do Estado do Paraná, entre 1990 e 1999, metade dos eventos de calamidades naturais esteve relacionado a alguma ocorrência de eventos meteorológicos severos (granizo, vendavais e tempestades). No Brasil houve um crescente aumento nos registros de desastres naturais relacionados ao clima desde a década de 70 , compondo $84 \%$ dos casos totais registrados.

Segundo Marcelino et al. (2005), a distribuição das ocorrências de desastres naturais por região do Brasil entre os anos de 1900 e 2006 revela que há uma tendência maior para a ocorrência desses fenômenos nas Regiões Sul, Sudeste e Nordeste, justamente as áreas mais habitados do país. Esse fato evidencia a necessidade de compreender melhor os fenômenos que geram esses eventos severos, conforme é sugerido por Nascimento (2005).

Observa-se a tendência à ocorrência de tempestades convectivas severas no Brasil por meio do trabalho desenvolvido por Romatschke e Houze (2010). Os autores desenvolveram um trabalho em que se buscou observar a profundidade dos núcleos convectivos que dão origem à precipitação na região de domínio correspondente a grande parte da América do Sul, durante o período de verão entre os anos de 1998 e 2006. Observou-se que a Região Sul do Brasil apresenta alta frequência de núcleos convectivos profundamente desenvolvidos, e essa instabilidade é alimentada também pelo Jato de baixos níveis que se estabelece na região, favorecendo o transporte de momento e umidade da região amazônica ao sul do Brasil. Nas regiões sudeste e centro-oeste do país também se observam intensos núcleos convectivos, os quais estão fortemente associas à Zona de Convergência da América do Sul que se estabelece naquela região. A região da Bacia Amazônica se caracteriza por ser amplamente coberta por núcleos convectivos menos profundos, entretanto muito mais frequentes, determinando o regime pluvial da região.

Dentre os fenômenos meteorológicos severos que oferecem grande risco à sociedade, destacam-se os downbursts. Trata-se de um fenômeno meteorológico, cujo termo foi cunhado por Fujita em 1985 e entende-se basicamente como uma forte coluna densa de ar frio, denominada por downdraft, que descende em direção ao solo e ao colidir-se induz a uma forte explosão de ventos divergentes, denominados de outburst.

Os perfis de vento e turbulência gerados por downbursts se apresentam diferentes do que se observa em sistemas de pressão amplamente desenvolvidos - Extended Pressure Systems (EPS). Dessa forma, há a preocupação do conhecimento específico desse fenômeno (especialmente em baixos níveis troposféricos) para a engenharia do vento, especialmente no que diz respeito à determinação das cargas de vento estabelecidas. Segundo Blesmann (1995), quando os projetos não visam ventos gerados por downbursts e outros fenômenos podem ocorrer acidentes, como destelhamentos, arrancamento de revestimentos e até mesmo colapsos estruturais de edificações leves ou com grandes vãos; além disso, esses ventos causam intenso desflorestamento (GARSTANG et al., 1998) e representam um risco à aviação, especialmente em momentos de aterrissagem e decolagem.

Caracena et al. (1989) sugere que os downbursts são fenômenos que podem ser normalmente confundidos com a ocorrência de tornados ou frentes de rajadas, entretanto eles costumam atingir menores áreas que os tornados e geram velocidades de vento mais intensas do que se verifica nas frentes de rajadas. Assim, verifica-se a necessidade em identificar os ambientes convectivos propícios à ocorrência de downbursts, pois, segundo Nascimento (2005), é necessário o aprimoramento de técnicas de previsão de tempestades convectivas severas no Brasil.

A ocorrência de downbursts no Brasil ainda é pouco explorada, as primeiras observações do fenômeno foram feitas nos anos noventa. Garstang et al. (1998) investigaram a ocorrência de downbursts na Bacia Amazônica e concluíram que a região centro-oeste daquela área apresenta padrões de quedas e torções de árvores decorrentes de downbursts úmidos. Na sequência, são observadas essas características também no extremo leste da região. Os autores concluíram que os máximos de precipitação coincidem com a distribuição dos blowdowns na floresta, registrados pelas imagens obtidas do Satélite Landsat.

Em 2000, Ponte (2005) cita um caso de downbursts que atingiram uma região metropolitana de Porto Alegre, que se estendia desde da zona oeste da capital até o município de Viamão - RS, e estimou-se que os ventos chegaram a $180 \mathrm{~km} / \mathrm{h}$. Lima e Menezes (2004) associaram em seus trabalhos danos em torres de transmissão com a ação de downbursts em Cachoeira Paulista - SP.

Em seis de novembro de 2008, no munícipio de Cachoeira Paulista, ocorreu um evento de downburst gerado a partir de um Sistema Convectivo de Mesoescala - SCM com ventos que chegaram a $37 \mathrm{~m} / \mathrm{s}$. O impacto dos danos foi tão intenso que levou a cidade a decretar estado de calamidade pública (CPTEC/INPE, 2008). 
Em 21 de julho de 2010 a estação meteorológica do INMET, localizada em Canela, registrou ventos de aproximadamente $35 \mathrm{~m} / \mathrm{s}$. Houve controvérsias na tentativa de definição do fenômeno, algumas fontes consideraram que os danos foram gerados a partir de downbursts (CLIC RBS, 2010), outras consideraram que houve a ação tornádica (BAND, 2010).

\section{Metodologia}

Na Tabela 1 são apresentados os valores dos índices CAPE e CINE, que podem indicar um ambiente atmosférico propício à formação de downbursts. Na Tabela 2 são apresentadas as variáveis meteorológicas e os seus valores característicos, que definem um ambiente atmosférico em que há a formação de downbursts.

Neste artigo serão apresentados dois estudos de casos. $\mathrm{O}$ primeiro visa analisar a relação existente entre altos valores do índice CAPE e CINE, com a ocorrência de variáveis meteorológicas que caracterizam a formação de um ambiente propício à ocorrência de downbursts. O segundo reflete a seleção de duas análises de tempo severo ocorridos nos estados do Rio Grande de Sul e Santa Catarina, em que se observou um padrão das variáveis meteorológicas semelhante ao que caracteriza a ocorrência de downbursts.

Tabela 1 - CAPE e CINE e seus respectivos valores mínimos que caracterizam ambientes instáveis e propícios à ocorrência de downbursts

\begin{tabular}{c|c|c}
\hline Características & $\begin{array}{c}\text { Valores que caracterizam } \\
\text { alta instabilidade atmosférica }\end{array}$ & Referências \\
\hline Índice CAPE (J/kg) & $1000-4000$ & $\begin{array}{c}\text { Prior (2007) } \\
\text { Nascimento (2005) } \\
\text { Caracena et al (1989) } \\
\text { Índice CINE (J/kg) }\end{array}$ \\
& $0<$ CINE $<50$ & \\
\hline
\end{tabular}

Tabela 2 - Intervalo de valores de variáveis meteorológicas que indicam a ocorrência de downbursts em ambientes tempestuosos

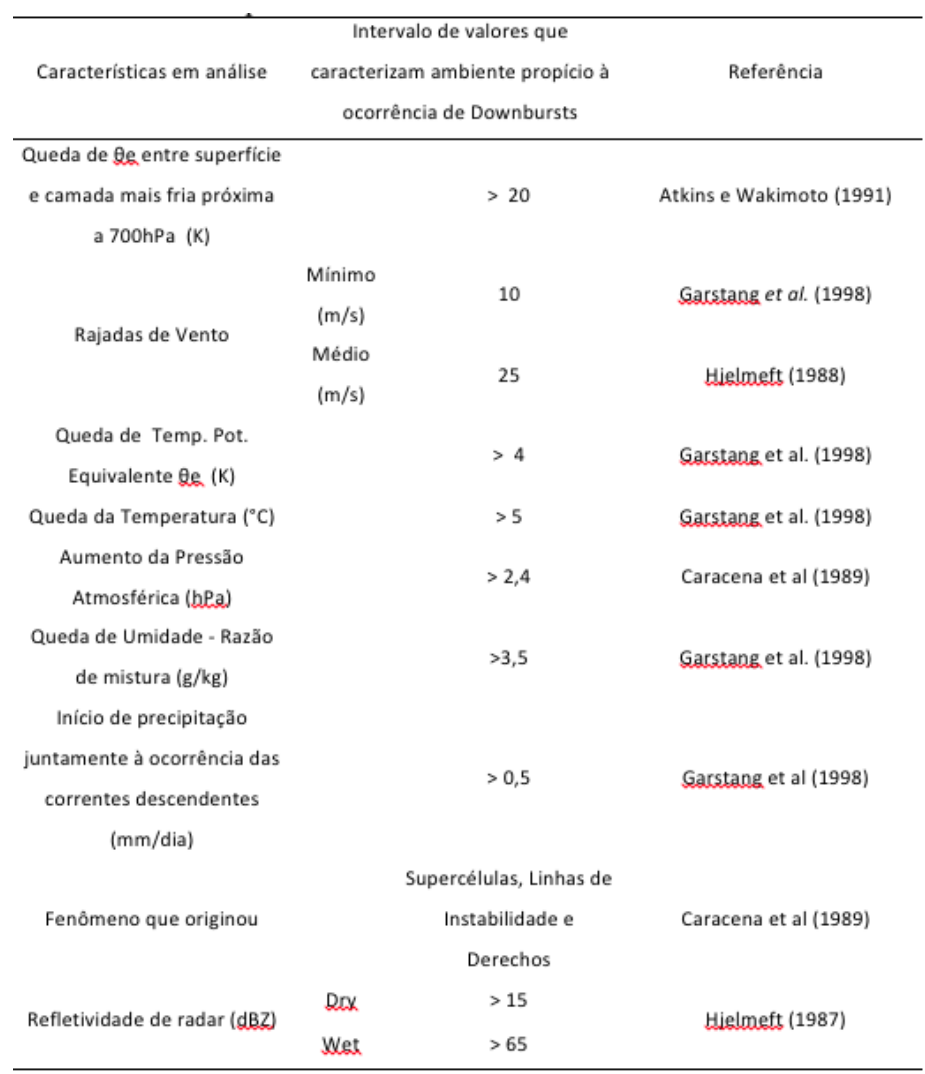


No primeiro estudo, foram selecionados 52 casos ocorridos em 26 localidades distribuídas ao longo Brasil. Esses casos apresentaram altos valores de índice CAPE e CINE, características essenciais, segundo Caracena et al. (1989) e Pryor (2007), para definir ambientes propícios à ocorrência de downbursts e, segundo Nascimento (2005), para caracterizar ambientes em que se verifica alta instabilidade, conforme a Tabela 1.

Os casos analisados compreendem o período do primeiro semestre do ano de 2013, os índices foram obtidos por meio das sondagens aerológicas, realizadas duas vezes ao dia nos locais de observação. Essas sondagens estão disponíveis ao acesso público por meio do site http://weather.uwyo.edu/upperair/sounding.html. Os dados meteorológicos horários foram obtidos a partir das Estações Meteorológicas Automáticas do Instituto Brasileiro de Meteorologia (INMET), localizadas nos locais ou próximas aos locais de lançamento das sondas aerológicas.

O segundo estudo de múltiplos casos compreende a observação das condições meteorológicas em dois casos de ocorrência de tempo severo na Região Sul do Brasil, ambos apresentam o desenvolvimento de linhas de instabilidade.

O primeiro caso analisado ocorreu durante a noite do dia 31 de dezembro de 2012, e o segundo ocorreu durante o dia 29 de maio de 2013, eles se caracterizaram especialmente pelos transtornos gerados à população dos locais mais atingidos. Para realizar essas observações, foram utilizados dados horários das Estações Meteorológicas Automáticas do INMET, espalhadas nos dois estados atingidos, além de sondagens aerológicas feitas nas cidades de Porto Alegre e Santa Maria, que fica a $252 \mathrm{~km}$ da capital. Os critérios obtidos a partir da revisão literária que evidenciam condições meteorológicas favoráveis a ocorrência de downbursts são apresentados na Tabela 2.

\section{Resultados}

Os dados obtidos, conforme visualizado na Tabela 3, revelam que apenas $15 \%$ dos casos que apresentaram um CAPE maior que $1000 \mathrm{~J} / \mathrm{kg}$ desenvolveram velocidades de rajadas superiores a $10 \mathrm{~m} / \mathrm{s}$, o mínimo proposto por Garstang et al. (1998) para a definição de ventos provenientes de downbursts; além disso houve precipitação em apenas $17 \%$ dos casos.

Percebe-se uma relação entre altos valores de $\Delta \theta$ e e índice CAPE, nos casos analisados, quando se verificam rajadas de vento com velocidades superiores a $10 \mathrm{~m} / \mathrm{s}$, os valores de CINE são baixos e os de CAPE altos.

No segundo estudo de caso, para analisar o comportamento das variáveis meteorológicas dos casos analisados, buscou-se identificar se elas definem padrões característicos pela ocorrência de downbursts. Para escolher as estações meteorológicas a ser estudado, o critério utilizado foi selecionar as estações que apresentaram valores de velocidade de vento maiores que $10 \mathrm{~m} / \mathrm{s}$.

Tabela 3 - Variáveis e seus respectivos valores mínimos que caracterizam ambientes propícios à ocorrência de downbursts

\begin{tabular}{|c|c|c|c|c|c|c|c|c|c|c|c|c|c|c|c|c|}
\hline \multicolumn{3}{|c|}{ Local } & \multicolumn{7}{|c|}{ Caso 1} & \multicolumn{7}{|c|}{ Caso 2} \\
\hline UF & Cidade & Cod. & Data & $\mathrm{Z}$ & $\begin{array}{l}\text { CAPE } \\
(\mathrm{J} / \mathrm{kg})\end{array}$ & $\begin{array}{c}\mathrm{CIN} \\
(\mathrm{J} / \mathrm{kg})\end{array}$ & $\begin{array}{c}\text { Vraj } \\
(\mathrm{m} / \mathrm{s})\end{array}$ & $\begin{array}{c}\Delta \theta \mathrm{e} \\
(\mathrm{k})\end{array}$ & $\begin{array}{l}\text { Prec. } \\
\text { (mm) }\end{array}$ & Data & $\bar{Z}$ & $\begin{array}{l}\text { CAPE } \\
(\mathrm{J} / \mathrm{kg})\end{array}$ & $\begin{array}{c}\mathrm{CIN} \\
(\mathrm{J} / \mathrm{kg})\end{array}$ & $\begin{array}{c}\text { Vraj } \\
(\mathrm{m} / \mathrm{s})\end{array}$ & $\begin{array}{c}\Delta \theta e \\
(\mathrm{k})\end{array}$ & $\begin{array}{l}\text { Prec. } \\
(\mathrm{mm})\end{array}$ \\
\hline AM & Manaus & A101 & $14 / 1$ & 0 & 1891 & 27 & 8,6 & 18,3 & 0,0 & $22 / 1$ & 0 & 1882 & 28 & 8,3 & 18,5 & 0,0 \\
\hline BA & Caravelas & A405 & $13 / 1$ & 12 & 1535 & 3 & 11,3 & 39,5 & 0,0 & $25 / 1$ & 12 & 1602 & 0 & 8,0 & 21,4 & 0,0 \\
\hline BA & Salvador & A401 & $19 / 1$ & 12 & 2370 & 55 & 6,4 & 20,8 & 0,0 & $20 / 1$ & 12 & 1445 & 60 & 7,5 & 25,2 & 0,0 \\
\hline CE & Fortaleza & A305 & $21 / 1$ & 12 & 1936 & 1 & 9,2 & 23,5 & 0,0 & $15 / 2$ & 12 & 2812 & 1 & 8,9 & 34,2 & 7,8 \\
\hline DF & Brasília & A045 & $10 / 4$ & 0 & 1650 & 19 & 4,2 & 11,5 & 0,0 & $15 / 4$ & 0 & 2026 & 17 & 7,5 & 23,5 & 0,0 \\
\hline ES & Vitória & A612 & $1 / 2$ & 0 & 1632 & 1 & 10,2 & 29,3 & 0,0 & $28 / 2$ & 0 & 1419 & 33 & 6,2 & 19,6 & 0,0 \\
\hline MA & Carolina & A205 & $4 / 3$ & 12 & 1861 & 22 & 7,9 & 36,5 & 0,0 & $27 / 3$ & 12 & 1839 & 20 & 5,3 & 28,3 & 0,0 \\
\hline MG & Confins & A521 & $2 / 2$ & 0 & 1494 & 24 & 5,3 & 9,0 & 0,0 & $19 / 1$ & 0 & 1626 & 38 & 6,3 & 13,7 & 2,2 \\
\hline MS & Campo Grande & A702 & $16 / 5$ & 12 & 1226 & 24 & 4,4 & 18,5 & 0,2 & $25 / 6$ & 0 & 1303 & 19 & 7,3 & 13,0 & 0,0 \\
\hline MT & Cuiabá & A901 & $18 / 1$ & 0 & 1794 & 26 & 7,2 & 29,5 & 0,0 & $14 / 2$ & 0 & 2167 & 36 & 5,8 & 26,3 & 0,0 \\
\hline PA & Belém & A201 & $15 / 3$ & 0 & 1954 & 5 & 8,1 & 25,0 & 9,4 & $3 / 2$ & 0 & 2575 & 2 & 11,1 & 17,7 & 5,4 \\
\hline PE & Recife & A301 & $18 / 1$ & 12 & 2311 & 0 & 2,7 & 24,7 & 0,0 & $8 / 2$ & 12 & 1616 & 2 & 8,0 & 28,5 & 0,0 \\
\hline $\mathrm{PE}$ & Petrolina & A307 & $27 / 1$ & 12 & 2239 & 12 & 8,5 & 30,2 & 0,0 & $26 / 1$ & 12 & 1658 & 8 & 6,0 & 24,2 & 0,0 \\
\hline $\mathrm{PI}$ & Floriano & A311 & $17 / 1$ & 12 & 1654 & 21 & 5,4 & 23,4 & 0,0 & $26 / 2$ & 12 & 1807 & 9 & 8,3 & 34,2 & 0,0 \\
\hline PR & Curitiba & A807 & $2 / 2$ & 0 & 1322 & 47 & 8,6 & 24,2 & 0,0 & $21 / 2$ & 0 & 1754 & 2 & 11,3 & 27,0 & 0,0 \\
\hline PR & Foz do Iguaçú & A846 & $1 / 5$ & 0 & 1887 & 78 & 13,5 & 24,8 & 1,6 & $1 / 6$ & 0 & 2902 & 54 & 9,5 & 29,8 & 0,0 \\
\hline RJ & Rio de Janeiro & A621 & $19 / 2$ & 12 & 2411 & 23 & 7,3 & 27,3 & 0,0 & $23 / 2$ & 0 & 2321 & 10 & 0,0 & 26,6 & 0,0 \\
\hline RN & Natal & A304 & $9 / 2$ & 0 & 2062 & 0 & 13,1 & 34,6 & 0,0 & $13 / 2$ & 0 & 2068 & 0 & 13,9 & 36,7 & 0,0 \\
\hline $\mathrm{RR}$ & Boa vista & A135 & $22 / 3$ & 0 & 1195 & 17 & 10,0 & 22,8 & 0,0 & $21 / 4$ & 0 & 1485 & 47 & 9,8 & 18,3 & 0,0 \\
\hline RS & Porto Alegre & A801 & $4 / 4$ & 0 & 1213 & 83 & 7,5 & 20,3 & 0,2 & $14 / 2$ & 0 & 1578 & 44 & 4,3 & 23,8 & 0,0 \\
\hline RS & Santa Maria & A803 & $17 / 2$ & 0 & 1582 & 71 & 5,9 & 30,8 & 0,0 & $4 / 4$ & 0 & 1621 & 18 & 5,9 & 19,2 & 0,0 \\
\hline SC & Florianópolis & A868 & $13 / 2$ & 0 & 1278 & 9 & 3,2 & 13,7 & 0,2 & $21 / 2$ & 12 & 1618 & 7 & 7,3 & 30,8 & 0,0 \\
\hline SP & São Paulo & A701 & $1 / 6$ & 12 & 4895 & 90 & 6,9 & 14,5 & 6,9 & $25 / 2$ & 0 & 2060 & 35 & 6,9 & 28,4 & 0,0 \\
\hline
\end{tabular}


A partir do momento em que se estabeleceu velocidade máxima instantânea $\left(\mathrm{V}_{\max }\right)$ para o período em que o complexo convectivo estudado atuou sobre a região analisada, foram tomadas relações específicas entre as variáveis observadas e estabeleceu-se para a análise a relação $\mathrm{V}_{\operatorname{med}} / \mathrm{V}_{\max }$ dada em percentagem, significando quanto a velocidade média horária representa em relação à velocidade máxima horária observada. Analisou-se também a variação entre a velocidade máxima e mínima horária $\left(\Delta \mathrm{T}_{\max \text {-min }}\right)$, pressão máxima e mínima horária $\left(\Delta \mathrm{P}_{\text {max-min }}\right)$, umidade específica máxima e mínima horária $\left(\Delta \mathrm{r}_{\text {max-min }}\right)$, temperatura potencial equivalente máxima e mínima horária $\left(\Delta \theta \mathrm{e}_{\max -\min }\right)$, umidade relativa máxima e mínima horária $\left(\Delta \mathrm{UR}_{\max -\min }\right)$ e a observação de precipitação no momento da rajada de vento máxima ou na hora seguinte, como é observado em dois casos indicados com $\left({ }^{*}\right)$. É ressaltado que a variação de umidade relativa não recebe considerações específicas nos textos analisados, pois a umidade específica representa mais precisamente a umidade atmosférica. A taxa de obtenção é de 3 segundos, e ocorre a uma altura padrão de $10 \mathrm{~m}$ acima do solo.

Por meio da aplicação dos critérios expostos na Tabela 2 , na Tabela 4 observa-se que $85 \%$ dos valores analisados estão acima do sugerido como mínimos pela literatura para definir a ocorrência de downbursts. Dessa forma, as rajadas de vento registradas podem ser provenientes deste fenômeno meteorológico em especial, e aquelas observadas em Jaguarão, em Dom Pedrito e em Rio
Grande, foram verificados valores de rajadas de vento que destoaram bastante da velocidade média observada naquela hora.

Ressalta-se que o índice CAPE e CINE registrados em Porto Alegre às 00Z do dia 1 de janeiro de 2013 foi de $1132 \mathrm{~J} / \mathrm{kg}$ e $137 \mathrm{~J} / \mathrm{kg}$, respectivamente, e para Santa Maria foi de $1648 \mathrm{~J} / \mathrm{kg}$ e 38,1 J/kg. Nesse caso, com exceção do índice CINE observado em Porto Alegre, os índices foram eficientes na caracterização das condições atmosféricas, sugerindo ambientes altamente instáveis.

Os dados obtidos no segundo estudo realizado são apresentados na Tabela 6, esse evento ocorreu no dia 29 de maio de 2013 , e percebe-se que $60 \%$ dos valores obtidos encontram-se acima do sugerido na literatura para caracterizar a ocorrência de downbursts. Este caso apresentou menos expressividade nas observações, entretanto, os dados registrados na estação do Morro da Igreja, em São Joaquim - SC, apresentam valores bastante próximos aos sugeridos pela literatura pra definir a ocorrência de downbursts, e rajada de vento com velocidades superiores a $100 \mathrm{~km} / \mathrm{h}$, em São José dos Ausentes - RS. Os valores obtidos também foram bastante expressivos, pois os índices CAPE e CINE registrados em Porto Alegre às $12 \mathrm{Z}$ deste mesmo dia foi de 1,61 J/ $\mathrm{kg}$ e $408 \mathrm{~J} / \mathrm{kg}$, respectivamente, e para Santa Maria foi de $275,1 \mathrm{~J} / \mathrm{kg}$ e $269 \mathrm{~J} / \mathrm{kg}$, respectivamente. Os valores menos significantes devem-se ao fato do fenômeno ter se estabelecido com maior intensidade no nordeste do Rio Grande do Sul e sul de Santa Catarina.

Tabela 4 - Dados obtidos no evento ocorrido na noite do dia 31 de dezembro de 2013 em diversas cidades do Rio Grande do Sul

\begin{tabular}{|c|c|c|c|c|c|c|c|c|c|c|}
\hline Local & Data & $\begin{array}{l}\text { Hora } \\
\text { (UTC) }\end{array}$ & $\begin{array}{l}\mathrm{V}_{\max } \\
\mathrm{m} / \mathrm{s}\end{array}$ & $\begin{array}{c}V_{\text {max }} / V_{\text {med }} \\
(\%)\end{array}$ & $\begin{array}{c}T_{\max }-T_{\min } \\
(\mathrm{K}) \\
\end{array}$ & $\begin{array}{c}P_{\max }-P_{\min } \\
(\mathrm{hPa})\end{array}$ & $\begin{array}{c}r_{\max }-r_{\min } \\
(\mathrm{g} / \mathrm{kg})\end{array}$ & $\begin{array}{c}\theta_{\mathrm{e} \text { max }}-\theta \mathrm{e}_{\min } \\
(\mathrm{K})\end{array}$ & $\begin{array}{c}U_{\text {Unax }}-U_{\text {min }} \\
(\%)\end{array}$ & $\begin{array}{l}\text { Prec. } \\
(\mathrm{mm})\end{array}$ \\
\hline Rio Grande & $1 / 1 / 13$ & 0000 & 18.70 & $47 \%$ & 6.40 & 4.30 & 3.6 & 17.35 & 24 & 28.6 \\
\hline Santa Maria & $1 / 1 / 13$ & 0200 & 17.90 & $8 \%$ & $\underline{10.50}$ & $\underline{5.40}$ & $\underline{17.6}$ & 23.45 & 33 & $\underline{34.4}$ \\
\hline $\begin{array}{l}\text { Santana do } \\
\text { Livramento }\end{array}$ & $12 / 31 / 12$ & 2300 & 21.80 & $33 \%$ & $\underline{2.20}$ & $\underline{4.60}$ & $\underline{14.6}$ & $\underline{21.45}$ & 30 & $\underline{20.2}$ \\
\hline Uruguaiana & $12 / 31 / 12$ & 2300 & 23.00 & $29 \%$ & $\underline{\underline{10.90}}$ & $\underline{\underline{4.40}}$ & $\underline{\underline{19.5}}$ & $\underline{28.92}$ & 38 & $\underline{\underline{176}}$ \\
\hline Canguçú & $1 / 1 / 13$ & 0100 & 22.40 & $32 \%$ & 0.40 & 2.40 & 0.6 & 0.87 & 2 & 23.2 \\
\hline $\begin{array}{l}\text { Caçapava } \\
\text { do Sul }\end{array}$ & $1 / 1 / 13$ & 0100 & 18.80 & $45 \%$ & 0.70 & 60 & 1.1 & 4 & 5 & $\underline{\underline{3}}$ \\
\hline Alegrete & $1 / 1 / 13$ & 0100 & 19.40 & $29 \%$ & 2.60 & $\underline{2.80}$ & $\underline{\underline{4.2}}$ & $\underline{\underline{5.62}}$ & 13 & $\underline{\underline{17}}$ \\
\hline Bagé & $12 / 31 / 12$ & 2300 & 19.20 & $22 \%$ & $\underline{\underline{8.80}}$ & $\underline{3.50}$ & $\underline{14.2}$ & $\underline{21.84}$ & 28 & $\underline{1.4}$ \\
\hline Quaraí & $12 / 31 / 12$ & 2300 & 28.80 & $24 \%$ & $\underline{\underline{9.60}}$ & $\underline{2.90}$ & $\overline{16.9}$ & $\underline{\underline{19.22}}$ & 36 & $\underline{\underline{19.8}}$ \\
\hline São Gabriel & $1 / 1 / 13$ & 0000 & 18.00 & $28 \%$ & $\underline{8.20}$ & 2.20 & $\underline{5.7}$ & $\underline{26.30}$ & 15 & Z4* \\
\hline Jaguarão & $12 / 31 / 12$ & 2200 & 16.00 & $45 \%$ & 3.50 & 2.80 & $\underline{5.6}$ & 2.34 & 14 & 11.4 \\
\hline Dom Pedrito & $12 / 31 / 12$ & 2300 & 19.00 & $70 \%$ & $\underline{\underline{8.00}}$ & $\underline{\underline{4.70}}$ & $\underline{13.1}$ & $\underline{24.84}$ & 25 & $22,6 *$ \\
\hline Chuí & $12 / 31 / 12$ & 2100 & 23.10 & $13 \%$ & $\underline{5.20}$ & $\underline{4.60}$ & $\underline{\underline{Z 5}}$ & $\underline{17.02}$ & 14 & 21.4 \\
\hline \multicolumn{11}{|c|}{ *Precipitação na hora seguinte } \\
\hline & & & 20.5 & $33 \%$ & 6.5 & 3.7 & 9.6 & 16.7 & 21.3 & 18.9 \\
\hline Desvio P & Padrão & & 3.2 & 0.2 & 3.5 & 1.0 & 6.4 & 9.2 & 11.2 & 8.3 \\
\hline
\end{tabular}


Tabela 5 - Dados obtidos no segundo estudo de múltiplos casos para o caso ocorrido no dia 29 de maio de 2013

\begin{tabular}{|c|c|c|c|c|c|c|c|c|c|c|}
\hline Local & Data & $\begin{array}{l}\text { Hora } \\
\text { (UTC) }\end{array}$ & $\begin{array}{l}\mathrm{V}_{\max } \\
\mathrm{m} / \mathrm{s}\end{array}$ & $\begin{array}{c}V_{\max } / V_{\text {med }} \\
(\%)\end{array}$ & $\begin{array}{c}T_{\max }-T_{\min } \\
(\mathrm{K})\end{array}$ & $\begin{array}{c}P_{\max }-P_{\min } \\
(\mathrm{hPa})\end{array}$ & $\begin{array}{c}r_{\max }-r_{\min } \\
(\mathrm{g} / \mathrm{kg})\end{array}$ & $\begin{array}{c}\theta e_{\max }-\theta e_{\min } \\
(\mathrm{K})\end{array}$ & $\begin{array}{c}U R_{\max }-U R_{\min } \\
(\%)\end{array}$ & $\begin{array}{l}\text { Prec. } \\
(\mathrm{mm})\end{array}$ \\
\hline \multirow{11}{*}{ RS } & Canela & $17: 00$ & 16.9 & $27 \%$ & 4.9.9 & 3.5 & 3.40 & 15.05 & 11 & $\underline{\underline{9.0}}$ \\
\hline & Cangucú & $22: 00$ & 20.7 & $37 \%$ & $\overline{4.7}$ & $\overline{2.9}$ & 2.48 & 11.73 & 2 & $\overline{10}$ \\
\hline & Uruguaiana & $11: 00$ & 21.5 & $35 \%$ & 3.2 & $\overline{8.4}$ & 1.71 & $\underline{2.53}$ & 8 & $\underline{10.6}$ \\
\hline & Rio Grande & $16: 00$ & 15.1 & $56 \%$ & 0.6 & 1.6 & 0.39 & 1.59 & 1 & $\underline{8.2}$ \\
\hline & Rio Pardo & $15: 00$ & 22.5 & $24 \%$ & 0.6 & $\underline{4.1}$ & 0.56 & 1.86 & 7 & $\underline{5.8}$ \\
\hline & Santa Maria & $14: 00$ & 25.4 & $40 \%$ & 4.5 & 4.3 & 3.27 & 13.95 & 11 & $\underline{\underline{4.0}}$ \\
\hline & Tramandaí & $17: 00$ & 23.6 & $20 \%$ & 2.1 & 4.8 & 1.59 & $\underline{6.33}$ & 3 & $\underline{12.6}$ \\
\hline & São Gabriel & $14: 00$ & 18.5 & $43 \%$ & 1.9 & 8.9 & 1.81 & $\underline{6.28}$ & 8 & $\underline{5.8}$ \\
\hline & Torres & $18: 00$ & 15.3 & $16 \%$ & 1.8 & 2.7 & 1.53 & $\underline{\underline{6.08}}$ & 4 & $\underline{\underline{76}}$ \\
\hline & Vacaria & $19: 00$ & 17.4 & $22 \%$ & 2.5 & $\overline{0.9}$ & 0.51 & $\overline{4.23}$ & 19 & $\overline{15.4}$ \\
\hline & Porto Alegre & $15: 00$ & 9.8 & $26 \%$ & 0.6 & $\underline{4.3}$ & 0.47 & $\overline{1.57}$ & 1 & 4.2 \\
\hline \multirow{8}{*}{ SC } & Araranguá & $19: 00$ & 17.8 & $14 \%$ & 3.4 & $\underline{4.7}$ & 1.23 & $\underline{\underline{6.83}}$ & 14 & $\underline{206}$ \\
\hline & $\begin{array}{c}\text { São José dos } \\
\text { Ausentes }\end{array}$ & $19: 00$ & 22.9 & $47 \%$ & $\underline{\underline{5.9}}$ & 1.5 & $\underline{3.62}$ & 17.19 & 13 & $\underline{\underline{11.0}}$ \\
\hline & São José & $23: 00$ & 11.6 & $31 \%$ & 3.6 & 2.7 & 1.50 & $\underline{\underline{8.01}}$ & 17 & $\underline{\underline{6.4}}$ \\
\hline & Urussanga & $21: 00$ & 14.5 & $8 \%$ & 4.1 & 1.7 & 2.04 & 10.21 & 28 & $\underline{\underline{0.6}}$ \\
\hline & Morro da Igreja & $21: 00$ & 30.6 & $64 \%$ & 4.3 & 1.7 & 2.93 & 13.34 & 5 & $\underline{\underline{12}}$ \\
\hline & Santa Marta & $20: 00$ & 31.7 & $20 \%$ & 4 & 1.9 & 0.80 & $\underline{\underline{6.59}}$ & 10 & $\underline{2.2}$ \\
\hline & São Joaquim & $21: 00$ & 18.5 & $26 \%$ & 0.7 & 1 & 0.41 & 1.86 & 7 & $\underline{3.8}$ \\
\hline & Ituporanga & $23: 00$ & 10.2 & $4 \%$ & 4.5 & 1.9 & 1.73 & $\underline{\underline{977}}$ & 30 & $\underline{\underline{8.6}}$ \\
\hline & Média & & 19.2 & 0.3 & 3.0 & 3.3 & 1.7 & 7.9 & 10.5 & 7.3 \\
\hline \multicolumn{2}{|c|}{ Desvio Padrão } & & 5.9 & 0.2 & 1.6 & 2.2 & 1.0 & 4.6 & 8.1 & 5.1 \\
\hline
\end{tabular}

\section{Conclusões}

Os casos analisados no primeiro estudo de caso apresentados revelam que a utilização dos índices de instabilidade de forma isolada não compreende uma estratégia eficiente para identificação de ambientes que podem dar origem à downbursts. Aconselha-se que, para uma completa caracterização da instabilidade atmosférica, sejam observados também outros índices meteorológicos.

Com o segundo estudo de caso é possível concluir, através dos padrões das variáveis observadas, que é possível observar a formação de downbursts no Brasil. Nos casos analisados, eles estiveram associados à passagem de linhas de instabilidade na Região Sul do país, e ressalta-se que elas não são exclusividade do sul do pais, podendo ocorrer no nordeste brasileiro devido à penetração dos ventos alísios sobre o continente e na região amazônica, além de que outros sistemas atuantes no Brasil podem gerar tempo severo.

Verifica-se a necessidade de uma completa caracterização da ocorrência de downbursts em território brasileiro, de maneira a contribuir com essa lacuna do conhecimento.

\section{Referências}

ATKINS, Nolan T.; ST. LAURENT, Michael. Bow echo mesovortices. Part I: Processes that influence their damaging potential. Monthly Weather Review, v. 137, n. 5, p. 1497-1513, 2009.
ATKINS, Nolan T.; WAKIMOTO, Roger M. Wet microburst activity over the southeastern United States: Implications for forecasting. Weather and forecasting, v. 6, n. 4, p. 470-482, 1991.

BAND. Tornado atingiu 300 moradias na Serra Gaúcha. 2010. Disponível em: noticias.band.uol.com.br/cidades/ noticia/?id=100000326569. Acessado em 17 de dezembro de 2013.

CARACENA, F.; HOLLE, R.; DOSWELL, C. Microbursts: a handbook for visual identificationLABORATORY. [S.1: s.n.]. Disponível em: <http://trid.trb.org/view. aspx?id=326028>. Acesso em: 17 abr. 2013.

CARACENA, Fernando; HOLLE, Ronald L.; DOSWELL, Charles A. Microbursts: a handbook for visual identification. LABORATORY, 1989.

CLICRBS. Após possível tornado na Serra, temperatura despenca e chuva pode chegar a $60 \mathrm{~mm}$ no Norte. Soledade - RS, 2013. Disponível em: www.clicsoledade.com.br/ clicnews/?pg=ler\&id=2968. Acesso 17 de dezembro de 2013.

CPTEC/INPE. Tempestade em Cachoeira Paulista (SP). 2008. Disponível em: www.cptec.inpe.br/ rupload/ arquivo/Cachoeira_061108.pdf॰. Acessado em 17 de dezembro de 2013.

FUJITA, Tetsuya Theodore. The downburst. SMRP, v. 210, p. 122, 1985. 
GARSTANG, M. et al. Convective cloud downdrafts as the cause of large blowdowns in the Amazon rainforest. Meteorology and Atmospheric Physics, v. 67, n. 1-4, p. 199-212, 1998.

GARSTANG, M. et al. Convective cloud downdrafts as the cause of large blowdowns in the Amazon rainforest. Meteorology and Atmospheric Physics, v. 67, n. 1-4, p. 199-212, 1998.

GUHA-SAPIR, Debarati. Disasters in numbers 2010. Center for the Epidemiology of Disasters.[Online], 2011.

HJELMFELT, Mark R. Structure and life cycle of microburst outflows observed in Colorado. Journal of Applied Meteorology, v. 27, p. 900-927, 1988.

HJELMFELT, Mark R. Structure and life cycle of microburst outflows observed in Colorado. Journal of applied meteorology, v. 27, n. 8, p. 900-927, 1988.

LIMA, D. R. O.; MENEZES, Wallace Figueiredo. Queda de estruturas de linha de transmissão de energia elétrica em Cachoeira Paulista (SP) e a ocorrência de tempestades severas-Um estudo de caso. In: CONGRESSO BRASILEIRO DE METEOROLOGIA, XIII. 2004.

LIMA, D. R. O.; MENEZES, Wallace Figueiredo. Queda de estruturas de linha de transmissão de energia elétrica em Cachoeira Paulista (SP) ea ocorrência de tempestades severas-Um estudo de caso. In: CONGRESSO BRASILEIRO DE METEOROLOGIA, XIII. 2004.

MARCELINO, Emerson Vieira. Desastres naturais e geotecnologias: conceitos básicos. Caderno Didático, v. 1, p. 34, 2007.

NASCIMENTO, E. DE L. Previsão de tempestades serveras Utilizando-se parâmetros convectivos e modelos de mesoescala: Uma estratégia operacional adotável no Brasil? Revista Brasileira de Meteorologia, v. 60, n. 1, p. 121-140, 2005.

NASCIMENTO, Ernani de Lima. Previsão de tempestades severas utilizando-se parâmetros convectivos e modelos de mesoescala: uma estratégia operacional adotável no Brasil. Revista Brasileira de Meteorologia, v. 20, n. 1, p. 121-140, 2005.

PRYOR, Kenneth L. The GOES Microburst Windspeed Potential Index. arXiv preprint physics/0703162, 2007.

ROMATSCHKE, Ulrike; HOUZE JR, Robert A. Extreme summer convection in South America. Journal of Climate, v. 23, n. 14, 2010. 